\title{
Electrically fueled active supramolecular materials
}

Serxho Selmani ${ }^{1,2}$, Eric Schwartz ${ }^{1,2}$, Justin T. Mulvey ${ }^{1,2}$, Hong Wei $^{1,3}$, Adam Grosvirt-Dramen ${ }^{1,4}$, Wyeth Gibson ${ }^{1,2}$, Allon I. Hochbaum ${ }^{1,2,3,4}$, Joseph P. Patterson ${ }^{1,2,3}$, Regina Ragan ${ }^{1,3}$, Zhibin Guan $^{1,2,3,4,6^{*}}$

${ }^{1}$ Center for Complex and Active Materials, University of California, Irvine, Irvine, California 92697, United States. ${ }^{2}$ Department of Chemistry, University of California, Irvine, Irvine, California 92697, United States. ${ }^{3}$ Department of Materials Science and Engineering, University of California, Irvine, Irvine, California 92697, United States. ${ }^{4}$ Department of Chemical and Biomolecular Engineering, University of California, Irvine, Irvine, California 92697, United States. ${ }^{5}$ Department of Molecular Biology and Biochemistry, University of California, Irvine, Irvine, California 92697, United States. ${ }^{6}$ Department of Biomedical Engineering, University of California, Irvine, Irvine, California 92697, United States. *e-mail: zguan@uci.edu

\begin{abstract}
:
Fuel-driven dissipative supramolecular assemblies in biology, such as actin filaments and microtubules contribute to the formation of complex, dynamic structures in living organisms and give rise to emergent functions such as motility, homeostasis, self-healing, and camouflage. Several synthetic dissipative supramolecular materials have been created using chemicals or light as fuel, with the goal of furthering our understanding of biological systems and creating synthetic materials that have life-like dynamic properties. However, electrical energy, one of the most common energy sources, has remained mostly unexplored for such purposes. Here we demonstrate the use of electrically fueled dissipative assembly as a new platform for creating active supramolecular materials. Through an electrochemical redox reaction network operating in mild aqueous buffers, a transient and highly active supramolecular assembly based on a redox-sensitive
\end{abstract}


cysteine derivative is achieved by applying an electric potential. The dissipative self-assembly as well as its emergent properties can be spatiotemporally controlled by modulation of electrical signals on patterned microelectrodes. Using electrical energy as a readily available and clean fuel, we are able to create dissipative supramolecular materials rapidly (in seconds to minutes) and repetitively under mild conditions with directional and spatiotemporal control. As electronic signals are the default information carriers in modern technology, the described approach offers a promising opportunity to integrate active materials into electronic devices for bioelectronics applications.

\section{INTRODUCTION}

Dissipative self-assemblies exhibit emergent properties far from equilibrium that are controlled spatiotemporally by the kinetics of fuel consumption ${ }^{1}$. Over the past decade, several synthetic dissipative assembly systems have been developed using chemical ${ }^{2-5}$ or light ${ }^{6-8}$ as the major fuel sources. Chemical fuels such as alkylating agents ${ }^{2}$, carbodiimides, ${ }^{9-10}$ ATP/GTP,,${ }^{11-15}$ and oxidants, ${ }^{16-18}$ have been used to drive dissipative assemblies of various building blocks including organic molecules, ${ }^{2,9-10,17,19}$ peptides, ${ }^{9,20-21}$ DNAs, ${ }^{15,}{ }^{22}$ and nanoparticles. ${ }^{23}$ Light has also been used to drive dissipative assembly of small molecules, ${ }^{24-25}$ proteins, ${ }^{7}$ and particles. ${ }^{6,26}$ These two main types of fueled active material systems complement each other with their respective advantages and limitations. ${ }^{3,8}$ Chemical fuels are versatile and have relatively high efficiency in generating the activated state, but face the challenge of generating waste in closed systems and are inherently limited in achieving spatiotemporal control of the dynamic assemblies. ${ }^{3}$ Light, on the other hand, is a cleaner fuel with no waste generation and can be delivered 
spatiotemporally to control the assembly process; however it is limited in penetration depth and has lower efficiency in generating the activated state. ${ }^{8}$

Another major energy form, electrical energy, has remained mostly unexplored for fueling active supramolecular assembly. Electrical energy offers several advantages as a fuel: it is readily available, clean, and can be modulated spatiotemporally. Electrical fields have been used to actuate electroactive polymers ${ }^{27}$ and liquid crystalline materials, ${ }^{28}$ control particle assembly, ${ }^{29}$ and power particle motions through various forms of electrostrictive hydrodynamics. ${ }^{30}$ Electric potential has also been shown to polymerize organic monomers ${ }^{31-32}$ and modulate supramolecular interactions. ${ }^{33-35}$ Here we report the first electrically fueled (e-fueled) dissipative self-assembly of active supramolecular materials in bulk solution.

\section{RESULTS AND DISCUSSION}

Designing e-fueled dissipative assembly system. To achieve our e-fueled dissipative selfassembly system, we designed an electrochemical redox reaction network that converts a cysteine derivative $(\mathrm{CSH})$ into its disulfide dimer (CSSC), which self-assembles into fibers (Fig. 1A). ${ }^{17,36}$ Ferrocyanide $\left(\mathrm{Fcn}^{4-}\right)$, a safe and biologically tolerated reagent, ${ }^{37}$ was used as a homogeneous electrocatalyst because it could be easily oxidized electrochemically to ferricyanide $\left(\mathrm{Fcn}^{3-}\right)$, which could further oxidize cysteine to the disulfide. ${ }^{38}$ The disassembly half of the redox reaction network was accomplished by using chemical reductant dithiothreitol (DTT). ${ }^{17}$ When a positive electric potential is applied to the working electrode, $\mathrm{Fcn}^{4-}$ near the electrode surface is oxidized to $\mathrm{Fcn}^{3-}$ via electron transfer to the electrode, which subsequently oxidizes $\mathrm{CSH}$ into CSSC 
initiating fiber growth from the surface (Fig. 1B). As the potential is removed, DTT present in solution reduces $\mathrm{CSSC}$ back to $\mathrm{CSH}$, resulting in the spontaneous dissolution of the fibers.
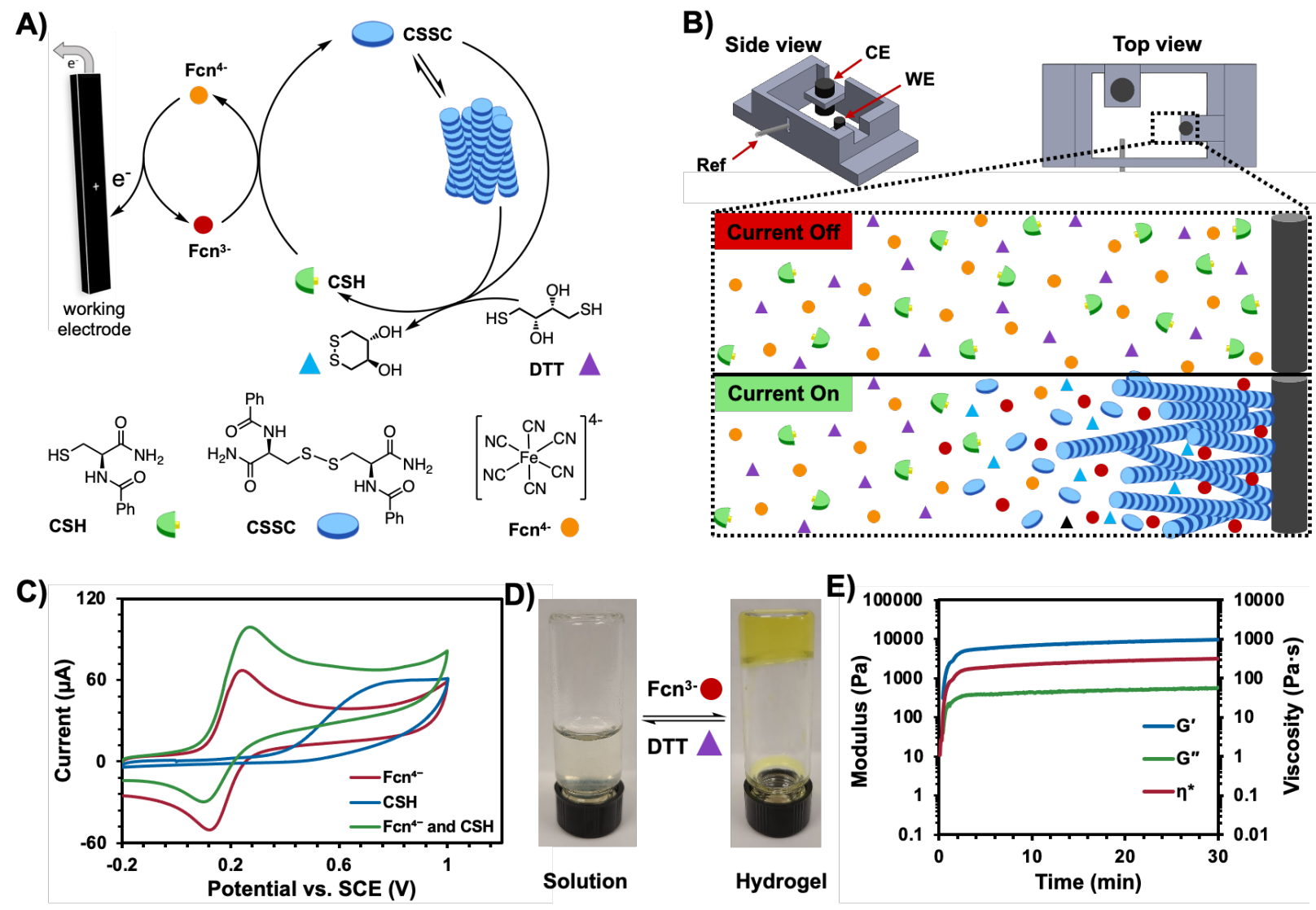

Figure 1. Design of electrically fueled active materials. A) Schematic representation of the dissipative self-assembly process fueled by electricity. B) Side and top view of the electrochemical cell used in the study $(\mathrm{CE}=$ glassy carbon counter electrode, $\mathrm{WE}=$ glassy carbon working electrode, Ref $=$ silver wire pseudo-reference electrode), and schematic representation of species distribution in the cell when current is turned on and off. C) Cyclic voltammogram showing electrocatalytic oxidation of CSH by ferricyanide $\left(\mathrm{Fen}^{3-}\right)$ generated in situ. D) Images showing the reversible fiber assembly and dissolution upon addition of $\mathrm{Fen}^{3-}$ or DTT to the solution $\left([\mathrm{CSH}]_{0}=5.0 \mathrm{mM}, \mathrm{pH}=8\right)$. E) Rheology measurements showing the formation of hydrogel upon addition of $\mathrm{Fen}^{3-}$ to $\mathrm{CSH}$ solution (same condition as $\mathbf{D}$ ).

To test the feasibility of our design, we first confirmed that $\mathrm{Fcn}^{4-}$ acts as a homogeneous electrocatalyst for $\mathrm{CSH}$ oxidation. Cyclic voltammograms $(\mathrm{CV})$ of $\mathrm{Fcn}^{4-}$ and $\mathrm{CSH}$ in aqueous buffer (Fig. 1C) shows that the former was easily and reversibly oxidized with a $E_{1 / 2}=200 \mathrm{mV}$ vs. $\mathrm{Ag} / \mathrm{Ag}^{+}$, whereas CSH was oxidized at $600 \mathrm{mV}$ vs. $\mathrm{Ag} / \mathrm{Ag}^{+}$irreversibly. When $\mathrm{Fcn}^{4-}$ was added to 
$\mathrm{CSH}$ solution, the $\mathrm{CV}$ became electrochemically irreversible as shown by the cathodic current being significantly smaller in magnitude than the anodic current, indicating that $\mathrm{Fcn}^{4-}$ was participating in the electrocatalytic oxidation of CSH. The kinetics for the redox reactions involved in the system were quantified through a combination of stopped flow UV-Vis spectroscopy and ultra-performance liquid chromatography (UPLC) (see SI for details). Based on the kinetics, we demonstrated that $\mathrm{Fcn}^{3-}$ could oxidize CSH to form CSSC gel, which could be dissolved by DTT, as was visualized by vial inversion (Fig. 1D) and confirmed by rheology (Fig. 1E, Fig. S14, Fig. S15).

e-Fueled transient and directional assembly. To demonstrate e-fueled dissipative selfassembly, we fabricated a custom glass-bottom, three-electrode bulk electrochemical cell (Fig. 1B, Fig. S1) in which e-fueled dissipative assembly could be monitored in situ using confocal laser scanning microscopy (CLSM). We screened experimental parameters to determine conditions for fast fiber growth and dissolution. We began by identifying the minimal $[\mathrm{CSH}]_{0}$ and maximal $[\mathrm{DTT}]_{0}$ that allowed for fiber formation at relatively low potential $\left(<1 \mathrm{~V} v \mathrm{Ag} / \mathrm{Ag}^{+}\right)$. Then, several experimental parameters were iteratively varied, including $\mathrm{pH},[\mathrm{CSH}]_{0},[\mathrm{DTT}]_{0}$, and electrical current. As expected, increasing the current led to faster and more vigorous fiber growth (Fig. S3) while increasing $[\mathrm{DTT}]_{0}$ accelerated the dissolution (Fig. S4). The kinetics of fiber dissolution were $\mathrm{pH}$ dependent, with higher $\mathrm{pH}$ resulting in faster dissolution (Fig. S5), in agreement with previous studies ${ }^{17}$. The concentration of $\mathrm{CSH}$ within the range of 2 to $5 \mathrm{mM}$ did not significantly impact growth and dissolution kinetics (Fig. S6). On the basis of these observations, the dissipative self-assembly was conducted in a pH 8 buffer with $2.5 \mathrm{mM} \mathrm{CSH}, 20 \mathrm{mM}$ DTT, $150 \mathrm{mM}$ ferrocyanide, and $25 \mu \mathrm{M}$ Nile red (as an intercalation dye for CLSM) ${ }^{17}$ by applying a potential of 
$430 \mathrm{mV}$ between the glassy carbon working electrode and silver wire pseudo reference electrode

(Fig. 1B).
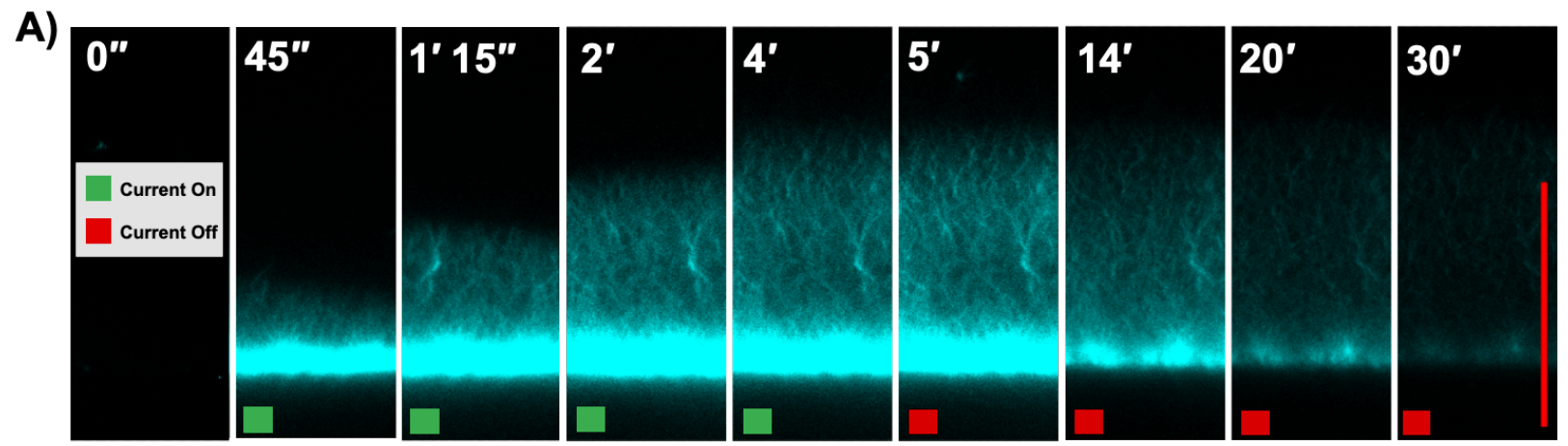

B)

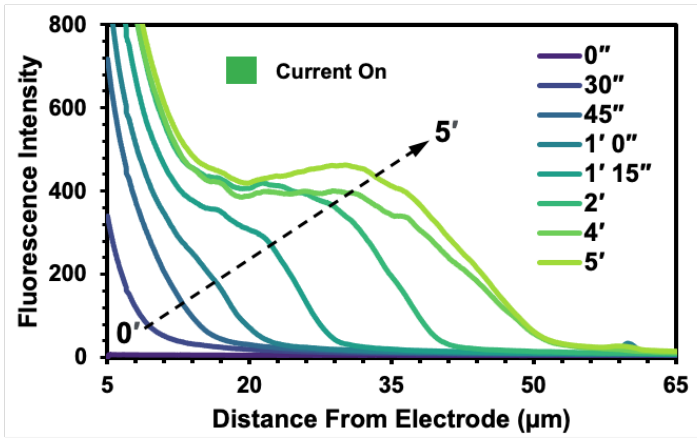

D)

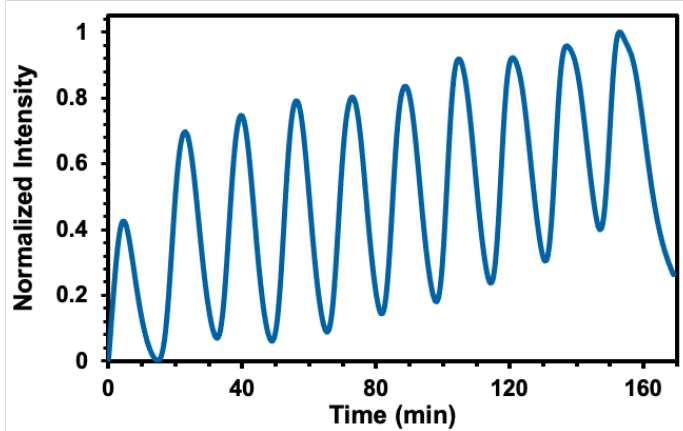

C)

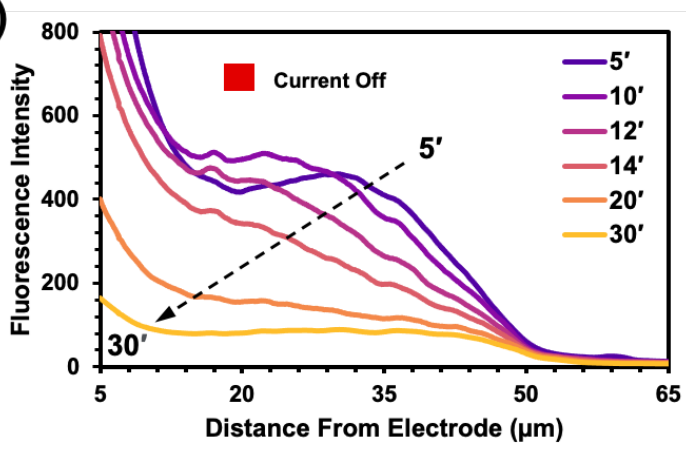

E)

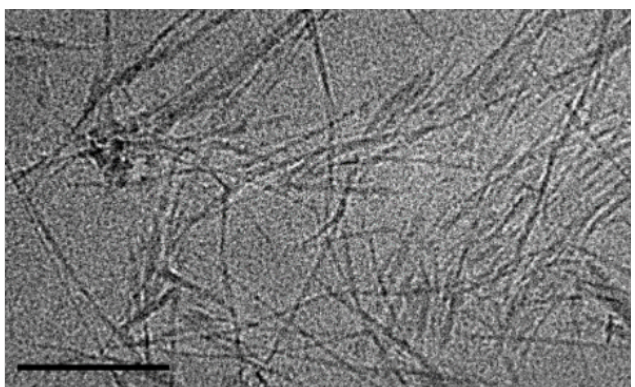

Figure 2. e-Fueled transient supramolecular assembly. A) CLSM snapshots showing directional fiber growth when current was applied (green square), and dissolution when current was turned off (red square) $\left(\mathrm{pH} 8,[\mathrm{CSH}]_{0}=2.5 \mathrm{mM},[\mathrm{DTT}]_{0}=20 \mathrm{mM},\left[\mathrm{Fcn}^{4-}\right]_{0}=150 \mathrm{mM}\right.$, Scale bar $=50 \mu \mathrm{m}, 175 \%$ hue saturation, electrode at bottom). B-C) Fluorescence intensity at varying distances from the electrode surface observed over time during fiber growth with current on $(\mathbf{B})$ and dissolution with current off $(\mathbf{C})$. D) Ten cycles of fiber growth and dissipation by repetitively switching current on and off $\left(600 \mathrm{mV} \mathrm{vs} \mathrm{Ag} / \mathrm{Ag}^{+}\right.$for $16 \mathrm{sec}$ followed by 24 min current off, under same condition as A). E) A cryo-TEM micrograph of self-assembled fibers formed by e-fueled assembly (Scale bar $=200 \mathrm{~nm}$ ). 
Shortly after applying the potential, fibers began to grow directionally perpendicular to the surface of the working electrode which continued to grow outwards when the potential was maintained (Fig. 2A, SI Movie 1). When the current was turned off at 5 minutes, the fibers began to disassemble and eventually disappear. The fiber morphology of the e-fueled assembly was confirmed by cryo-TEM imaging, showing fibers with average diameters $\sim 12 \mathrm{~nm}$ and average length $\sim 412 \mathrm{~nm}$ (Fig. 2E, Fig. S17). The fibers were observed to stack and coil together creating larger micron sized assemblies (Fig. S16). The fluorescence histograms of the image reveal that during the fiber growth with the current on, they grew outwards as a front while steadily increasing in fluorescence intensity throughout the body of the gel (Fig. 2B). Conversely, when the fibers began to disassemble with the current off, the fiber front gradually receded toward the electrode while the fluorescence intensity dropped across the gel as a whole (Fig. 2C). This is consistent with the fact that as the ferricyanide $\left(\mathrm{Fen}^{3-}\right)$ is generated at the electrode, it transports away from the electrode and oxidizes CSH into CSSC at the fiber front, enabling fibers to grow outwards (Fig. 1B). Upon depletion of the ferricyanide when current is turned off, the DTT in the bulk solution will diffuse back into the gel, initially reducing fibers at the front but then the body of the gel. Furthermore, the transient fibers could be grown and dissolved repetitively by turning the current on and off (Fig. 2D). These experiments clearly demonstrate the transient nature, directional growth, and precise temporal control of the e-fueled dissipative self-assembly.

Active and dynamic self-assembly. Next, we investigated active, dynamic self-assembly for the $\mathrm{CSH} / \mathrm{CSSC}$ system fueled by electricity. To observe the dynamics on a shorter time scale, we replaced DTT with a more efficient reductant, (tris(2-carboxyethyl)phosphine) (TCEP). ${ }^{39}$ The efueled system provides a convenient way to tune the kinetics of the reaction in real time by 
modulating the potential applied to the system. After applying an initial potential of $2 \mathrm{Vvs}$. $\mathrm{Ag} / \mathrm{Ag}^{+}$ for 11 second to grow a layer of fibers from the electrode surface, the potential was reduced to 600 $\mathrm{mV}$ to slow down the fiber growth for observing dynamics at the fiber front. The CLSM video shows highly complex active, dynamic processes for the system (SI Movie 2), especially at the fiber-water interface (Fig. 3A,B). The selected snapshots highlight different modes of activity for the self-assembly of fibers, including simultaneously growing and shrinking (Fig. 3C), waving (Fig. 3D), and curling and looping (Fig. 3E). Another observation is that as new fibers form at the front they collapse onto the body of the fibers already formed (SI Movie 2). The complexity of the observed active behavior could be attributed to multiple processes potentially ongoing in the system. In addition to the anticipated dissipative assembly/disassembly, several physicochemical processes may influence the active behavior. The electrochemical reaction should create concentration gradients for both the redox-sensitive species and electrolytes near the working electrode surface (Fig. 1B), which may cause liquid-liquid phase separation of the growing fibers into a gel phase. ${ }^{40}$ In addition, various electrostrictive hydrodynamics, such as electrohydrodynamics and/or electro-osmosis, ${ }^{41-42}$ may also contribute to the complex dynamic behavior.

The self-assembly dynamics observed in CLSM (SI Movie 2) were quantified using the structural dissimilarity index measurement (DSSIM) (Fig. 3B, F). ${ }^{43}$ DSSIM is a standard measure of the difference between two images, which compares variation in the mean, variance, and crosscorrelation between local regions of two images (see SI for details). DSSIM images were obtained by comparing two frames taken 90 seconds (Fig. 3B, S9, SI Movie 3) or 4 seconds (Fig. S9-10, SI Movie 3) apart in a sliding-window calculation. The 90 second sliding-window calculation quantifies dynamics occurring over a large time window while the 4 seconds sliding-window 
calculation captures the high temporal resolution dynamics. Each of the 240 DSSIM images were divided into five equally spaced regions with increasing distance from the working electrode, and the average DSSIM in each region is plotted for every frame (Fig. 3F, Fig. S10d, SI Movie 3).
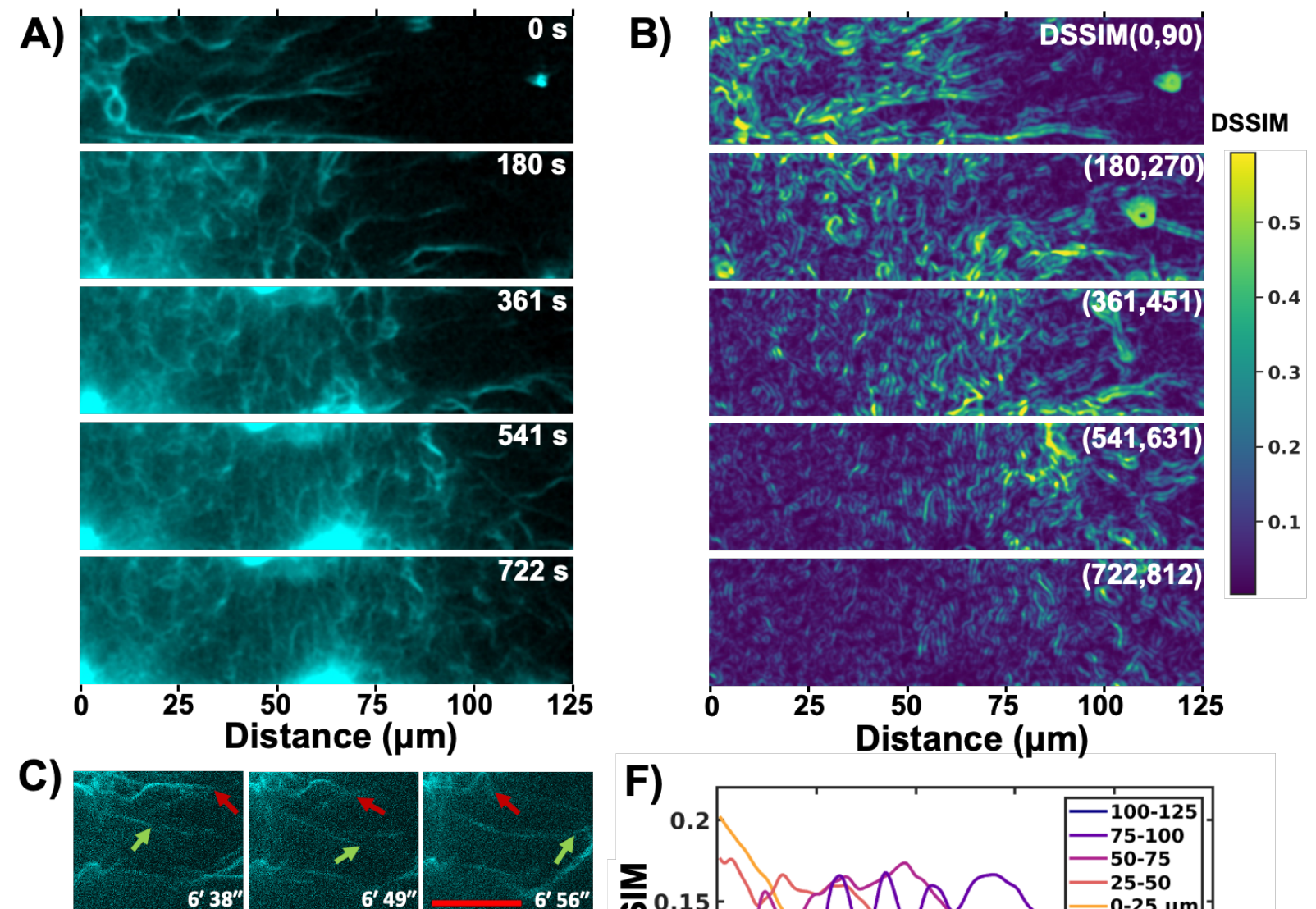

D)
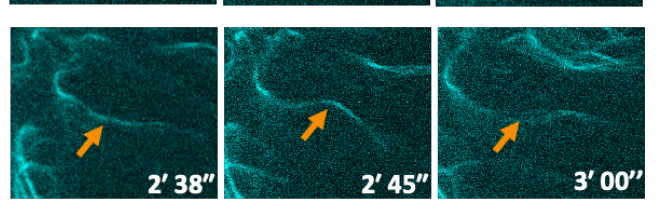

E)
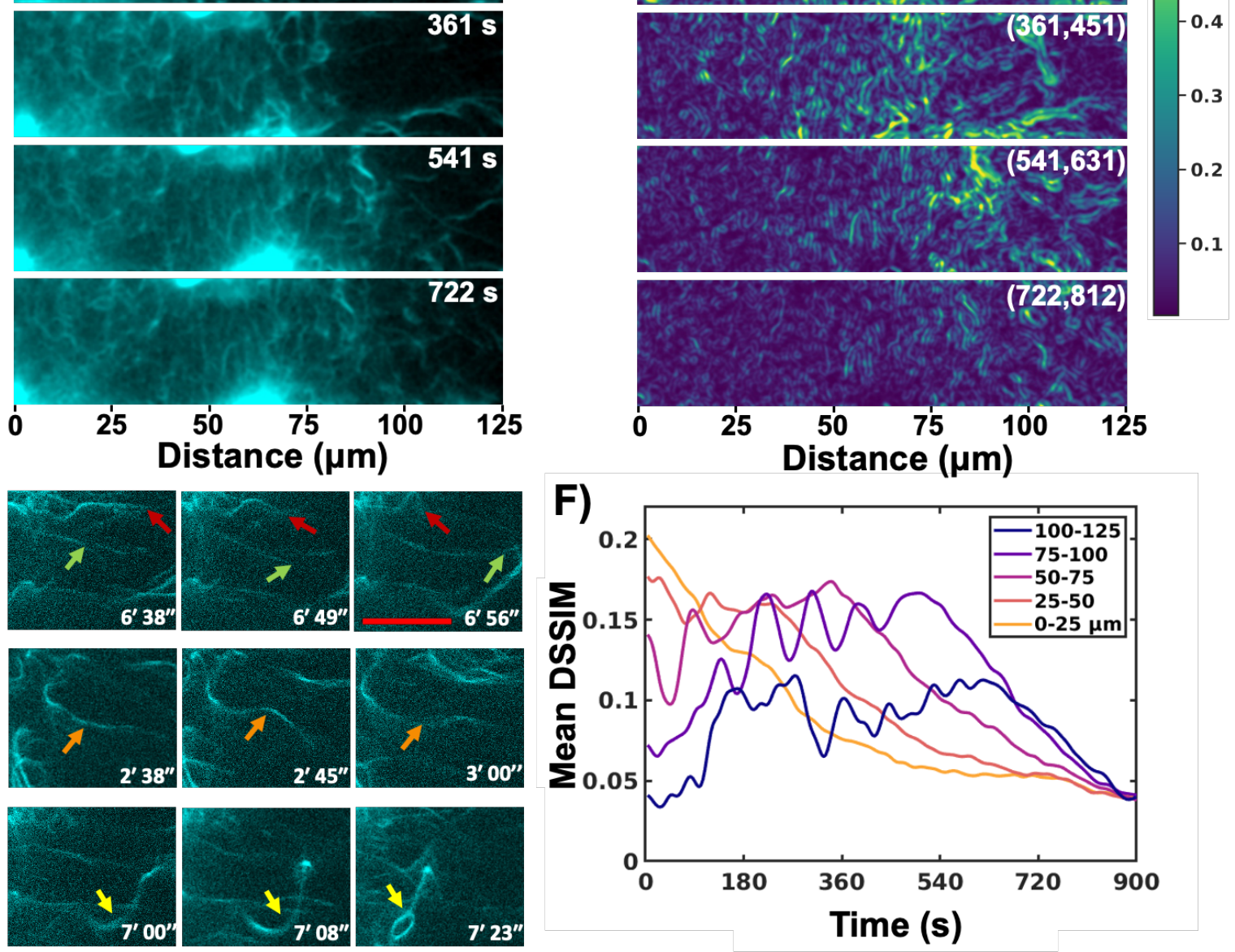

Figure 3. Active dynamic self-assembly fueled by electricity. A) Processed CLSM snapshots highlighting the high dynamics for self-assembly at the gel front (details in SI). B) Structural dissimilarity frame series generated by calculating the DSSIM of CLSM frames taken $90 \mathrm{~s}$ apart. Bright pixels represent regions of high structural dissimilarity between local areas in frames of comparison, which corresponds to fiber dynamics. C-E) Snapshots showing different active fiber movements: simultaneous fiber growth and shrinkage (C, green arrow for growing fiber and red for shrinking), waving (D), and curling/looping (E) (CLSM images rendered with gamma $=0.45$ and $150 \%$ hue saturation for fiber visualization). F) Mean DSSIM in each region seen in $\mathbf{B}$. (the working electrode for $\mathbf{A}-\mathbf{E}$ is out of frame on the left) 
The results show that the dissipative self-assembly system is highly dynamic with the most dynamic changes at the fiber-water interface which is seen for both the 90 seconds and 4 seconds intervals. The dynamics decrease with distance from the fiber front, as can be seen with the timedelayed decline in DSSIM values starting with the $0-25 \mu \mathrm{m}$ region which is then echoed in the 2550 and 50-75 $\mu \mathrm{m}$ regions. The 75-100 and 100-125 $\mu \mathrm{m}$ regions start with low DSSIM values which steadily increase as the fiber-water interface progresses further from the electrode until a peak is reached, and then gradually decline. The data also shows that the dynamics at the fiber-water interface are highest in the early stages and gradually decrease over time.

Spatiotemporal control of active assembly. Spatiotemporal control is a hallmark of biological dissipative self-assembly systems which is critical for their emergent functions. To demonstrate spatiotemporal control of the e-fueled active material system, we designed an array of interdigitated gold microelectrodes for use in a two-electrode configuration (Fig. 4A and Fig. S2). Each of the five bottom electrodes are individually addressable working electrodes, whereas the top five are connected as a single counter/reference electrode. By placing the sample solution (2.5 $\mathrm{mM} \mathrm{CSH}, 150 \mathrm{mM}$ ferrocyanide, $60 \mathrm{mM}$ DTT, $\mathrm{pH} 8$ buffer) on the interdigitated microelectrodes and applying $2.4 \mathrm{~V}$ vs. ref for 90 seconds, fibers were grown at each individually addressed electrode (Fig. 4B). When the current was turned off, it took roughly 90 seconds to fully dissolve the fibers and return to baseline fluorescence. By selectively applying electric potential to different working electrodes over time, while also varying the duration for current on and off, the first four measures of Beethoven's "Ode to Joy" were "played" on the microelectrode array (Fig. 4C, Fig. S7, SI Movie 4). This experiment clearly demonstrates precise spatiotemporal control and fast assembly/disassembly kinetics for the e-fueled dissipative system. 
A)

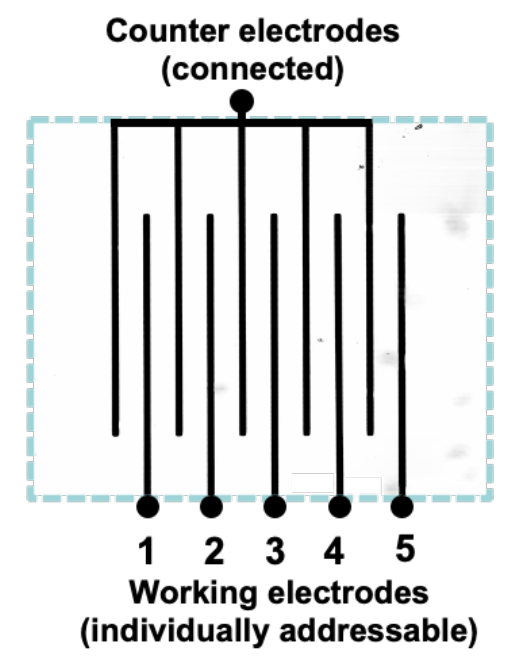

B)
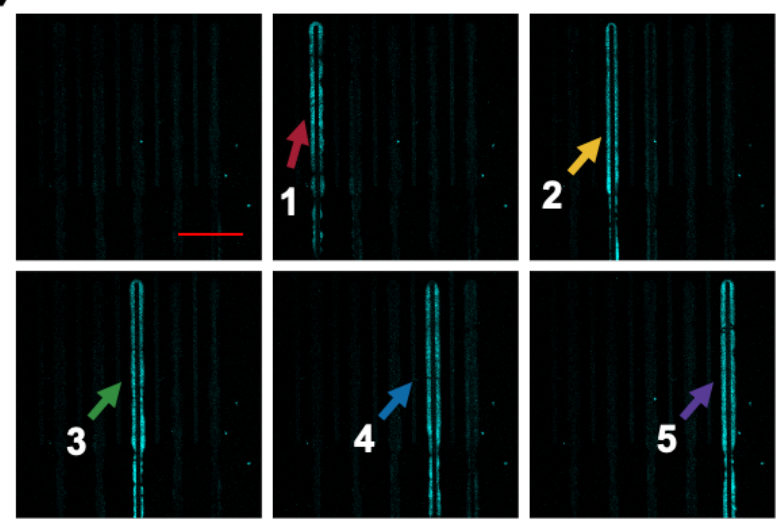

C)

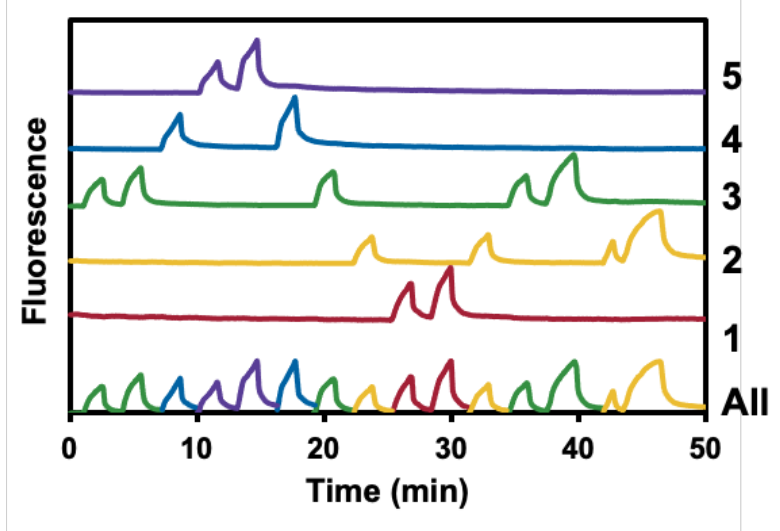

D)

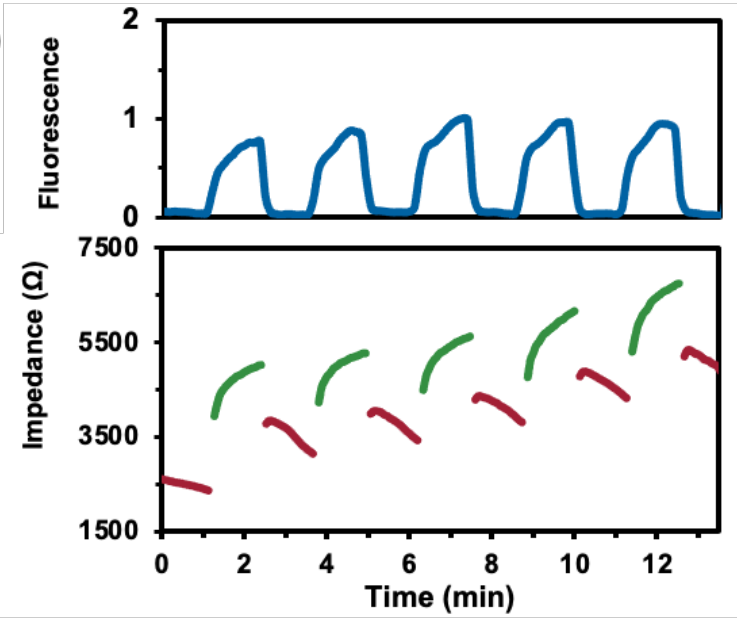

Figure 4. Spatiotemporal control of e-fueled dissipative assembly. A) Schematic representation of the individually addressable interdigitated gold microelectrodes. B) Growth of fibers at each of the working electrodes in A by selectively applying electric potential at different times. (CLSM images rendered at $150 \%$ hue saturation) C) Normalized fluorescence intensity measured around each working electrode to monitor fiber growth pattern after selectively applying electric potential to each electrode at different times for varying durations. Each line corresponds to the working electrode of matching number and colour in $\mathbf{B}$. The bottom trace superimposes 1-5 into one. If placed on a treble staff with the red graph at middle C, the pattern mimics that of the first four measures of Beethoven's "Ode to Joy" (see Fig. S7 and SI Movie 4). D) Fluorescence and impedance changes in the system upon cycling fiber growth at $2.4 \mathrm{~V}$ and fiber dissolution at $0 \mathrm{~V}$ over 5 cycles.

We also demonstrated that the e-fueled dissipative self-assemblies could dynamically transduce electronic inputs related to sensing, actuation, and computation. Single-frequency electrochemical impedance spectroscopy (SFEIS) was used to probe the impedance changes of the 
e-fueled dissipative assembly system on carbon-coated interdigitated gold microelectrodes (Fig. $\mathrm{S} 2)$ at $8 \mathrm{kHz}$. The impedance of the system increased sharply upon application of $2.4 \mathrm{~V} \pm 10 \mathrm{mV}$ vs. ref for one minute, resulting from the growth of fiber networks between electrodes, and dropped after the potential was returned to $0 \mathrm{~V} \pm 10 \mathrm{mV}$ vs. $\operatorname{ref}($ Fig. 4D). The fiber growth was concurrently monitored using CLSM during SFEIS measurements, confirming that the impedance changes correlate with fiber assembly and disassembly as indicated by changes in fluorescence intensity (Fig. 4D). In contrast, the control system without $\mathrm{CSH}$ in the solution only showed minimal changes of impedance under identical conditions (Fig. S13). The increase in impedance is attributed to the inhibition of ionic currents between electrodes due to diminished ionic transport through the self-assembled fiber network.

\section{CONCLUSION}

Here we have demonstrated a versatile new platform for dissipative self-assembly of active materials by using an electric current as the fuel. Electrical inputs are able to rapidly and repetitively fuel the dissipative self-assembly of fibers with directionality, high dynamics, and precise spatiotemporal control. These combined features would be difficult to be achieved with either chemical or light fueled dissipative systems. Furthermore, the system operates in mild aqueous buffers, uses electrical energy as a clean fuel and works at relatively low voltage. These features make this scheme ideal for developing sustainable dissipative self-assembly systems as well as applying this design to other redox sensitive assembling building blocks and/or microelectrode arrays. In addition, electronic signals fueling dissipative assembly are promising for interfaces with biological systems at cellular and tissue levels, as exemplified by the classical patch-clamp system and cardiac pacemakers. As electronic signals are at the heart of many modern 
technologies, the described e-fueled active material platform may find potential applications in sensors, prosthetics, soft robotics, neuromorphic computing and tissue engineering. ${ }^{44-45}$

\section{ACKNOWLEDGEMENTS}

This work was financially supported by the US Department of Energy, Office of Science, Basic Energy Sciences (DE-FG02-04ER46162; Z.G.; development of e-fueled dissipative self-assembly in bulk solution) and the National Science Foundation Materials Research Science and Engineering Center program through the UC Irvine Center for Complex and Active Materials (DMR-2011967; Z.G., A.I.H., J.P.P., R.R.; demonstration of e-fueled self-assembly on microelectrodes and comprehensive characterizations of e-fueled dissipative systems by CLSM, cryo-TEM, DSSIM analysis, and electrochemical impedance spectroscopy), with partial support from the National Science Foundation (CHE-1904939; Z.G.; kinetic study). The authors also acknowledge the use of facilities and instrumentation at the UC Irvine Materials Research Institute (IMRI) supported in part by the National Science Foundation Materials Research Science and Engineering Center program through the UC Irvine Center for Complex and Active Materials (DMR-2011967). H.W. acknowledges the use of facilities and instrumentation at the Integrated Nanosystems Research Facility (INRF) in the Samueli School of Engineering at the University of California Irvine. This study was made possible in part through access to the Optical Biology Core Facility of the Developmental Biology Center, a shared resource supported by the Cancer Center Support Grant (CA-62203) and Center for Complex Biological Systems Support Grant (GM076516) at the University of California, Irvine. S.S. acknowledges support by an NSERC postdoctoral fellowship from the Research Council of Canada. J.T.M. acknowledges the donors of 
The American Chemical Society Petroleum Research Fund. The authors thank Shane Ardo and Jenny Yang for help with electrochemical studies.

\section{REFERENCES:}

1. Nicolis, G.; Prigogine, I., Self-Organization in Nonequilibrium Systems: From Dissipative Structures to Order Through Fluctuations. 1977; p 506 pp.

2. Boekhoven, J.; Hendriksen, W. E.; Koper, G. J. M.; Eelkema, R.; van Esch, J. H., Transient assembly of active materials fueled by a chemical reaction. Science 2015, 349 (6252), 1075-1079.

3. Riess, B.; Groetsch, R. K.; Boekhoven, J., The Design of Dissipative Molecular Assemblies Driven by Chemical Reaction Cycles. Chem 2020, 6 (3), 552-578.

4. Das, K.; Gabrielli, L.; Prins, L. J., Chemically Fueled Self-Assembly in Biology and Chemistry. Angew. Chem., Int. Ed. 2021, doi.org/10.1002/anie.202100274.

5. Mattia, E.; Otto, S., Supramolecular systems chemistry. Nat. Nanotech. 2015, 10 (2), 111-119.

6. Klajn, R.; Wesson, P. J.; Bishop, K. J. M.; Grzybowski, B. A., Writing Self-Erasing Images using Metastable Nanoparticle "Inks". Angew. Chem., Int. Ed. 2009, 48 (38), 7035-7039.

7. Ross, T. D.; Lee, H. J.; Qu, Z.; Banks, R. A.; Phillips, R.; Thomson, M., Controlling organization and forces in active matter through optically defined boundaries. Nature 2019, 572 (7768), 224229.

8. Weissenfels, M.; Gemen, J.; Klajn, R., Dissipative Self-Assembly: Fueling with Chemicals versus Light. Chem 2021, 7 (1), 23-37.

9. Tena-Solsona, M.; Riess, B.; Groetsch, R. K.; Loehrer, F. C.; Wanzke, C.; Kaesdorf, B.; Bausch, A. R.; Mueller-Buschbaum, P.; Lieleg, O.; Boekhoven, J., Non-equilibrium dissipative supramolecular materials with a tunable lifetime. Nat. Commun. 2017, 8, 15895.

10. Kariyawasam, L. S.; Hartley, C. S., Dissipative Assembly of Aqueous Carboxylic Acid Anhydrides Fueled by Carbodiimides. Journal of the American Chemical Society 2017, 139 (34), 11949-11955.

11. Sorrenti, A.; Leira-Iglesias, J.; Sato, A.; Hermans Thomas, M., Non-equilibrium steady states in supramolecular polymerization. Nat. Commun. 2017, 8, 15899.

12. Maiti, S.; Fortunati, I.; Ferrante, C.; Scrimin, P.; Prins, L. J., Dissipative self-assembly of vesicular nanoreactors. Nat. Chem. 2016, 8 (7), 725-731.

13. Mishra, A.; Korlepara, D. B.; Kumar, M.; Jain, A.; Jonnalagadda, N.; Bejagam, K. K.; Balasubramanian, S.; George, S. J., Biomimetic temporal self-assembly via fuel-driven controlled supramolecular polymerization. Nat. Commun. 2018, 9 (1), 1-9.

14. te Brinke, E.; Groen, J.; Herrmann, A.; Heus, H. A.; Rivas, G.; Spruijt, E.; Huck, W. T. S., Dissipative adaptation in driven self-assembly leading to self-dividing fibrils. Nat. Nanotech. 2018, $13(9), 849-855$.

15. Heinen, L.; Walther, A., Programmable dynamic steady states in ATP-driven nonequilibrium DNA systems. Sci. Adv. 2019, 5 (7), eaaw0590.

16. Leira-Iglesias, J.; Tassoni, A.; Adachi, T.; Stich, M.; Hermans, T. M., Oscillations, travelling fronts and patterns in a supramolecular system. Nat. Nanotech. 2018, 13, 1021-1027.

17. Ogden, W. A.; Guan, Z., Redox Chemical-Fueled Dissipative Self-Assembly of Active Materials. ChemSystemsChem 2020, 2 (4), e1900030.

18. Colomer, I.; Morrow Sarah, M.; Fletcher Stephen, P., A transient self-assembling self-replicator. Nat. Commun. 2018, 9 (1), 2239. 
19. Bal, S.; Das, K.; Ahmed, S.; Das, D., Chemically Fueled Dissipative Self-Assembly that Exploits Cooperative Catalysis. Angew. Chem., Int. Ed. 2019, 58 (1), 244-247.

20. Debnath, S.; Roy, S.; Ulijn, R. V., Peptide Nanofibers with Dynamic Instability through Nonequilibrium Biocatalytic Assembly. J. Am. Chem. Soc. 2013, 135 (45), 16789-16792.

21. Kubota, R.; Makuta, M.; Suzuki, R.; Ichikawa, M.; Tanaka, M.; Hamachi, I., Force generation by a propagating wave of supramolecular nanofibers. Nat. Commun. 2020, 11 (1), 3541.

22. Del Grosso, E.; Prins, L. J.; Ricci, F., Transient DNA-Based Nanostructures Controlled by Redox Inputs. Angew. Chem., Int. Ed. 2020, 59 (32), 13238-13245.

23. Groetsch, R. K.; Wanzke, C.; Speckbacher, M.; Angi, A.; Rieger, B.; Boekhoven, J., Pathway Dependence in the Fuel-Driven Dissipative Self-Assembly of Nanoparticles. J. Am. Chem. Soc. 2019, 141 (25), 9872-9878.

24. Ikegami, T.; Kageyama, Y.; Obara, K.; Takeda, S., Dissipative and Autonomous Square-Wave Self-Oscillation of a Macroscopic Hybrid Self-Assembly under Continuous Light Irradiation. Angew. Chem., Int. Ed. 2016, 55 (29), 8239-8243.

25. de Jong, J. J. D.; Hania, P. R.; Pugzlys, A.; Lucas, L. N.; de Loos, M.; Kellogg, R. M.; Feringa, B. L.; Duppen, K.; van Esch, J. H., Light-driven dynamic pattern formation. Angew. Chem., Int. Ed. 2005, 44 (16), 2373-2376.

26. Klajn, R.; Bishop, K. J. M.; Grzybowski, B. A., Light-controlled self-assembly of reversible and irreversible nanoparticle suprastructures. Proc. Nat. Acad. Sci. U.S.A. 2007, 104 (25), 1030510309.

27. Bar-Cohen, Y., Electroactive Polymer (EAP) Actuators as Artificial Muscles: Reality, Potential, and Challenges, Second Edition. 2004.

28. Woltman, S. J.; Jay, G. D.; Crawford, G. P., Liquid-crystal materials find a new order in biomedical applications. Nat. Mater. 2007, 6 (12), 929-938.

29. Yeh, S.-R.; Seul, M.; Shraiman Boris, I., Assembly of ordered colloidal aggregrates by electricfield-induced fluid flow. Nature 1997, 386 (6620), 57-59.

30. Ma, F.; Yang, X.; Zhao, H.; Wu, N., Inducing propulsion of colloidal dimers by breaking the symmetry in electrohydrodynamic flow. Phys. Rev. Lett. 2015, 115 (20), 208302/1-208302/5.

31. Cosnier, S.; Karyakin, A.; Editors, Electropolymerization: Concepts, Materials And Applications. 2010; p 280 pp.

32. Ellis, T. K.; Galerne, M.; Armao, J. J. I. V.; Osypenko, A.; Martel, D.; Maaloum, M.; Fuks, G.; Gavat, O.; Moulin, E.; Giuseppone, N., Supramolecular Electropolymerization. Angew. Chem., Int. Ed. 2018, 57 (48), 15749-15753.

33. Chen, S.; Itoh, Y.; Masuda, T.; Shimizu, S.; Zhao, J.; Ma, J.; Nakamura, S.; Okuro, K.; Noguchi, H.; Uosaki, K.; Aida, T., Subnanoscale hydrophobic modulation of salt bridges in aqueous media. Science 2015, 348 (6234), 555-559.

34. Krabbenborg, S. O.; Veerbeek, J.; Huskens, J., Spatially Controlled Out-of-Equilibrium Host-Guest System under Electrochemical Control. Chem. Eur. J. 2015, 21 (27), 9638-9644.

35. Raeburn, J.; Alston, B.; Kroeger, J.; McDonald, T. O.; Howse, J. R.; Cameron, P. J.; Adams, D. J., Electrochemically-triggered spatially and temporally resolved multi-component gels. Mater. Horizons 2014, 1 (2), 241-246.

36. Otto, S.; Furlan, R. L. E.; Sanders, J. K. M., Selection and amplification of hosts from dynamic combinatorial libraries of macrocyclic disulfides. Science 2002, 297 (5581), 590-593.

37. Tschirhart, T.; Kim, E.; McKay, R.; Ueda, H.; Wu, H.-C.; Pottash, A. E.; Zargar, A.; Negrete, A.; Shiloach, J.; Payne, G. F.; Bentley, W. E., Electronic control of gene expression and cell behaviour in Escherichia coli through redox signalling. Nat. Commun. 2017, 8, 14030.

38. Nekrassova, O.; Allen, G. D.; Lawrence, N. S.; Jiang, L.; Jones, T. G. J.; Compton, R. G., The oxidation of cysteine by aqueous ferricyanide: a kinetic study using boron doped diamond electrode voltammetry. Electroanalysis 2002, 14 (21), 1464-1469.

39. Burns, J. A.; Butler, J. C.; Moran, J.; Whitesides, G. M., Selective reduction of disulfides by tris(2carboxyethyl)phosphine. J. Org. Chem. 1991, 56 (8), 2648-50. 
40. Saini, S.; Bukosky, S. C.; Ristenpart, W. D., Influence of Electrolyte Concentration on the Aggregation of Colloidal Particles near Electrodes in Oscillatory Fields. Langmuir 2016, 32 (17), 4210-4216.

41. Ristenpart, W. D.; Jiang, P.; Slowik, M. A.; Punckt, C.; Saville, D. A.; Aksay, I. A., Electrohydrodynamic Flow and Colloidal Patterning near Inhomogeneities on Electrodes. Langmuir 2008, 24 (21), 12172-12180.

42. Davidson Scott, M.; Andersen Mathias, B.; Mani, A., Chaotic induced-charge electro-osmosis. Phys. Rev. Lett. 2014, 112 (12), 128302.

43. Wang, Z.; Bovik Alan, C.; Sheikh Hamid, R.; Simoncelli Eero, P., Image quality assessment: from error visibility to structural similarity. IEEE transactions on image processing : a publication of the IEEE Signal Processing Society 2004, 13 (4), 600-12.

44. Song, E.; Li, J.; Won, S. M.; Bai, W.; Rogers, J. A., Materials for flexible bioelectronic systems as chronic neural interfaces. Nat. Mater. 2020, 19 (6), 590-603.

45. Liu, Y.; Li, J.; Song, S.; Kang, J.; Tsao, Y.; Chen, S.; Mottni, V.; McConnell, K.; Xu, W.; Zheng, Y.-Q.; Tok, J. B. H.; George, P. M.; Bao, Z., Morphing electronics enable neuromodulation in growing tissue. Nat. Biotech. 2020, 38 (9), 1031-1036. 